\title{
Prediction of generalization of ocular myasthenia gravis under immunosuppressive therapy in Northwest China
}

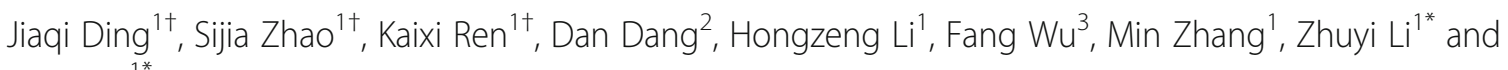
Jun GuO ${ }^{1 *}$ (D)

\begin{abstract}
Background: It is well demonstrated that immunosuppressants can reduce, but not eliminate the risk of generalized development in ocular myasthenia gravis (OMG). In this study, we aimed to explore the predictive factors of generalized conversion of OMG patients who received immunosuppressive treatments.

Methods: OMG patients under immunosuppressive treatments in Tangdu Hospital from June 2008 to June 2012 were retrospectively reviewed. Baseline clinical characteristics were documented. Patients were followed up regularly by face-to-face interview and the main outcome measure was generalized conversion. The logistic regression analysis was performed to determine the predictive factors of generalization of OMG.

Results: Two hundred twenty-three eligible OMG patients completed the final follow-up visit and 38 (17.0\%) progressed to generalized $M G(G M G)$ at a median time to generalization of 0.9 year. Patients with adult onset and positive repetitive nerve stimulation (RNS) of facial or axillary nerve had higher conversion rate than those with juvenile onset and negative RNS ( $p=0.001 ; p=0.019 ; p=0.015$, respectively). Adult-onset patients converted earlier than juvenile-onset OMG patients $(p=0.014)$. Upon multivariate logistic regression analysis, age of onset (Odds ratio [OR] 1.023, 95\% confidence interval [CI] 1.006-1.041, $p=0.007$ ) and positive facial nerve RNS (OR 2.826, 95\%Cl $1.045-5.460, p=0.038$ ) were found to be positively associated with generalized development. Moreover, an obviously negative association was found for disease duration (OR 0.603, 95\% Cl 0.365-0.850, $p=0.019$ ).

Conclusions: Age of onset, disease duration and facial nerve RNS test can predict generalized conversion of OMG under immunosuppressive therapy. Adult-onset, shorter disease duration and facial nerve RNS-positive OMG patients have a higher risk of generalized development.
\end{abstract}

Keywords: Myasthenia gravis, Ocular, Generalization, Predictive factor, Immunosuppressive therapy

\footnotetext{
*Correspondence: guojun_81@163.com; lizhuyi@fmmu.edu.cn

${ }^{\dagger}$ Jiagi Ding, Sijia Zhao and Kaixi Ren contributed equally to this work.

'Department of Neurology, Tangdu Hospital, Fourth Military Medical University, Xi'an 710038, Shaanxi Province, China

Full list of author information is available at the end of the article
}

(c) The Author(s). 2020 Open Access This article is licensed under a Creative Commons Attribution 4.0 International License, which permits use, sharing, adaptation, distribution and reproduction in any medium or format, as long as you give appropriate credit to the original author(s) and the source, provide a link to the Creative Commons licence, and indicate if changes were made. The images or other third party material in this article are included in the article's Creative Commons licence, unless indicated otherwise in a credit line to the material. If material is not included in the article's Creative Commons licence and your intended use is not permitted by statutory regulation or exceeds the permitted use, you will need to obtain permission directly from the copyright holder. To view a copy of this licence, visit http://creativecommons.org/licenses/by/4.0/ The Creative Commons Public Domain Dedication waiver (http://creativecommons.org/publicdomain/zero/1.0/) applies to the data made available in this article, unless otherwise stated in a credit line to the data. 


\section{Background}

Myasthenia gravis (MG) is an acquired neuromuscular junction disorder mainly mediated by pathogenic autoantibodies against acetylcholine receptors at the postsynaptic membrane. According to clinical manifestations, MG is classified as ocular MG (OMG) and generalized MG (GMG). Prior studies have shown that approximately $70 \%$ of MG patients initially present pure ocular symptoms and more than $90 \%$ of OMG patients undergo generalized conversion within 2-3 years [1, 2]. When systemic involvement of skeletal muscles is present, a diagnosis of GMG can be made and its outcome becomes worse [3-5]. Nowadays, it has been widely accepted that generalization of OMG is a classical phenomenon even though the conversion rate varies in different studies, and a series of predictors have been postulated to be correlated with secondary generalization. In Korean and Singapore populations, repetitive nerve stimulation (RNS) tests and antiacetylcholine receptor antibody (AChR-Ab) have been identified as predictive factors for generalization of OMG [6, 7]. While in a Germany study, the presence of thymoma was deemed to be the only risk factor for secondary generalization [8]. Previous studies have suggested that immunosuppressive therapy reduce the conversion rate of OMG to $7-23.3 \%[6,7,9]$, but a proportion of patients still ineluctably experience secondary generalization, and the predictors of generalization in these patients require further investigation. This study attempts to explore the potential predictors of generalized conversion in OMG patients under immunosuppressive therapy.

\section{Methods}

\section{Design, population and settings}

A total of 223 OMG patients under immunosuppressive treatments in Tangdu Hospital from June 2008 to June 2012 were retrospectively reviewed. The OMG diagnosis was confirmed in a double-blinded manner by two attending physicians or higher according to initial symptoms of unilateral or bilateral ptosis, diplopia, or both and at least one of the followings: (1) unequivocal response to neostigmine, (2) positive RNS tests, and (3) seropositivity for AChR-Ab if available. The exclusion criteria included: (1) GMG symptoms concurrent with the onset of OMG or within the first month of disease [10], (2) neonatal myasthenia gravis [11], and (3) disagreed clinical diagnoses were presumed by two attending physicians or higher. All patients were treated with steroids alone or in association with other immunosuppressants including azathioprine, immunoglobulin and cyclosporine A (Table 1). Steroid treatments included intravenous glucocorticoid pulse therapy and oral prednisone at an initial dose of $0.75-1.0$ $\mathrm{mg} / \mathrm{kg}$ per day with slow tapering according to clinical
Table 1 Demographic features of 223 OMG patients

\begin{tabular}{|c|c|}
\hline Variables & $n(\%)$ \\
\hline Disease duration (y), median (IQR) & $4(2-6)$ \\
\hline Gender & $(N=223)$ \\
\hline Male & $116(52.0)$ \\
\hline Female & $107(48.0)$ \\
\hline Age of onset & $(N=223)$ \\
\hline$<20 y$ & $110(49.3)$ \\
\hline$\geq 20 y$ & $113(50.7)$ \\
\hline Initial symptoms & $(N=223)$ \\
\hline Unilateral ptosis & $110(49.3)$ \\
\hline Bilateral ptosis & $40(17.9)$ \\
\hline Diplopia & $10(4.5)$ \\
\hline Unilateral ptosis with diplopia & $42(18.8)$ \\
\hline Bilateral ptosis with diplopia & $21(9.4)$ \\
\hline Neostigmine test & $(N=171)$ \\
\hline Positive & $163(95.3)$ \\
\hline Negative & $8(4.7)$ \\
\hline \multicolumn{2}{|l|}{ Positive rate of RNS test } \\
\hline Facial nerve & 25/84 (29.8) \\
\hline Axillary nerve & $18 / 76(23.7)$ \\
\hline Ulnar nerve & $2 / 74(2.7)$ \\
\hline Thyroid abnormality & $(N=102)$ \\
\hline Hyperthyroidism & $9(8.8)$ \\
\hline Subclinical hyperthyroidism & $4(3.9)$ \\
\hline Hypothyroidism & $2(2.0)$ \\
\hline Subclinical hypothyroidism & $2(2.0)$ \\
\hline Thymus abnormality & $(N=174)$ \\
\hline Thymoma & $25(14.4)$ \\
\hline Thymic hyperplasia & $7(4.0)$ \\
\hline Non-degraded thymus & $7(4.0)$ \\
\hline Immunosuppressants & $(N=223)$ \\
\hline Steroids & $159(71.3 \%)$ \\
\hline Azathioprine & $8(3.6 \%)$ \\
\hline Steroids + azathioprine & $53(23.8 \%)$ \\
\hline Steroids + cyclosporine A & $2(0.9 \%)$ \\
\hline Steroids + IVlg & $1(0.4 \%)$ \\
\hline
\end{tabular}

Abbreviations: RNS Repetitive nerve stimulation, y Year, IQR Interquartile range, IVIg Intravenous immunoglobulin. Unless otherwise noted, values are shown as $n(\%)$

condition. Intravenous immunoglobulin was administered at $0.4 \mathrm{~g} / \mathrm{kg}$ per day for 5 consecutive days. Oral azathioprine was given at $50 \mathrm{mg}-100 \mathrm{mg}$ per day and cyclosporine $\mathrm{A}$ at $100 \mathrm{mg}$ per day.

\section{Study protocol and data collection}

OMG patients' medical records were retrospectively reviewed and face-to-face interviews were conducted 
after written informed consent was obtained. Clinical variables including gender, age at onset, disease duration, clinical symptoms, response to neostigmine and RNS tests, thymus and thyroid examination were collected. When describing demographic features of the OMG patients, disease duration was defined as the interval from ocular symptom onset to the last follow-up irrespective of the presence of generalized conversion. In the last follow-up visit, a comprehensive interview was conducted to determine whether or not generalized conversion had occurred, which was defined as appearance of any systemic symptoms beyond extraocular muscle weakness such as dysphagia, dysarthria or weakness of extremities and even respiratory difficulties. Meanwhile, time to generalization was determined in the patients undergoing generalized conversion and re-identified as disease duration for those patients during the following logistic regression analysis for exploring the predictors of generalization.

\section{Statistical analysis}

Data was presented as number with percentage or median with interquartile range (IQR) and statistical analysis was performed by SPSS19.0 software (SPSS Inc., Chicago, IL, USA). Differences of categorical variables between groups were evaluated by $\chi^{2}$ test and Fisher's exact test when necessary. Manne-Whitney $U$ test was used to analyze the difference in age of onset and generalized interval between groups. Probability of generalized conversion was presented using the Kaplan-Meier method and analyzed with log-rank test. Univariate logistic regression analysis was performed on variables of disease duration, age at onset, electrophysiological tests and thymic abnormalities. Multivariate logistic regression analysis was performed using variables with $p<$ 0.100 during the univariate analysis to find the predictors of generalization of OMG. Odds ratio (OR) with 95\% confidence intervals (CI) was calculated. A $p$ value $<0.05$ was considered statistically significant in all tests.

\section{Results}

\section{Demographic features of OMG patients}

A total of 223 OMG patients who completed the final follow-up were included in this retrospective study. The study population consisted of 116 males and $107 \mathrm{fe}$ males, with a male-to-female ratio of 1.1:1. The median disease duration was 4 years (IQR, 2-6 years). The percentage of juvenile-onset patients $(<20$ y) was $49.3 \%$ and of adult-onset patients $(\geq 20 \mathrm{y})$ was $50.7 \%$ (Table 1 ). There were no significant differences in initial symptoms and complications including positive rates of thymoma between juvenile-onset and adult-onset patients ( $p>$ $0.05)$. The positive rate of neostigmine test of the two groups was $94.9 \%$ in juvenile-onset group and $95.7 \%$ in adult-onset group $(p=0.799)$. In juvenile-onset group, 23, 24 and 31 cases were examined with facial, axillary and ulnar RNS tests, respectively. And 61, 52 and 44 cases were examined with facial, axillary and ulnar RNS tests respectively in adult-onset group. While only the difference in the positive rate of axillary RNS test between the two groups showed statistical significance $(p=0.032)$ (Table 2).

\section{Conversion of OMG to GMG}

Thirty-eight out of 223 OMG patients (17.0\%; 18 males and 20 females) progressed to GMG with a median interval of 0.9 years (IQR, $0.3-2.1$ years). The median age of onset was 46 years (IQR, 37.5-55 years) in male patients and 30.5 years (IQR, 8-49.8 years) for female patients, and no significant difference was seen between the two groups $(p=0.118)$. Among the 38 patients undergoing generalization, 21 (55.3\%; 8 males and 13 females) progressed to type IIA according to the Osserman classification. Nine male and 6 female patients conversed to type IIB. The remaining 1 male and $1 \mathrm{fe}$ male patients progressed to type III. No significant difference was observed in the distribution of conversion types between the two groups $(p=0.512)$ (Table 3$)$.

\section{Probability of conversion to GMG}

Kaplan-Meier method was used to obtain cumulative probabilities for conversion from OMG to GMG. No significant difference was observed between males and females $(p=0.606)$, but a significantly higher probability of conversion to GMG was found in adult-onset OMG patients than juvenile-onset OMG patients $(p=0.001)$. Similarly, patients with positive facial or axillary nerve RNS tests had a significantly higher probability of conversion to GMG than those with negative RNS tests $(p=0.019$ and 0.015 , respectively) (Fig. 1). We further assessed the intervals from OMG onset to conversion in the 38 patients undergoing generalization. Adult-onset patients had an obviously shorter time to generalization than juvenile-onset patients $(p=0.014)$, but no significant differences were observed between male and female patients $(p=0.749)$, patients with positive and negative facial nerve RNS tests $(p=0.094)$ and patients with positive and negative axillary nerve RNS tests $(p=0.733)$, respectively (Fig. 2).

\section{Predictors of conversion to GMG}

Clinical variables including gender, age at onset, disease duration, positive facial or axillary nerve RNS and thymic abnormalities were selected for univariate logistic regression analysis to explore the potential predictors of generalization. Among these, age at onset (Odds ratio [OR] 1.025; 95\% confidence interval [CI] 1.009-1.041; $p=0.002$ ), disease duration (OR 0.783 ; 95\%CI $0.651-$ 
Table 2 Comparison of clinical features between juvenile-onset and adult-onset OMG patients

\begin{tabular}{|c|c|c|c|}
\hline Variables & $<20 y$ & $\geq 20 y$ & $P$ value \\
\hline Gender (Male/Female) & $110(53 / 57)$ & $113(63 / 50)$ & 0.258 \\
\hline \multicolumn{4}{|l|}{ Initial symptoms } \\
\hline Unilateral ptosis & $58 / 110(52.7 \%)$ & $52 / 113(46.0 \%)$ & 0.316 \\
\hline Bilateral ptosis & 20/110 (18.2\%) & 20/113 (17.7\%) & 0.925 \\
\hline Diplopia & $2 / 110(1.8 \%)$ & 8/113 (7.1\%) & 0.102 \\
\hline Unilateral ptosis with diplopia & 18/110 (16.4\%) & $24 / 113(21.2 \%)$ & 0.352 \\
\hline Bilateral ptosis with diplopia & $12 / 110(10.9 \%)$ & $9 / 113(8.0 \%)$ & 0.452 \\
\hline Neostigmine test $(+)$ & 74/78 (94.9\%) & 89/93 (95.7\%) & 0.799 \\
\hline \multicolumn{4}{|l|}{ Positive rate of RNS test } \\
\hline Facial nerve & $10 / 23(43.5 \%)$ & 15/61 (24.6\%) & 0.091 \\
\hline Axillary nerve & $2 / 24(8.3 \%)$ & $16 / 52(30.8 \%)$ & 0.032 \\
\hline Ulnar nerve & $0 / 31$ & $2 / 44(4.5 \%)$ & - \\
\hline Thyroid abnormality & $5 / 42(11.9 \%)$ & $12 / 60(20.0 \%)$ & 0.280 \\
\hline Hyperthyroidism & $2 / 5(40 \%)$ & $7 / 12(58.3 \%)$ & 0.620 \\
\hline Subclinical hyperthyroidism & $2 / 5(40 \%)$ & $2 / 12(16.7 \%)$ & 0.538 \\
\hline Hypothyroidism & $1 / 5(20 \%)$ & $1 / 12(8.3 \%)$ & 0.515 \\
\hline Subclinical hypothyroidism & $0 / 5$ & $2 / 12(16.7 \%)$ & - \\
\hline Thymus abnormality & 12/76 (15.8\%) & 27/98 (27.6\%) & 0.065 \\
\hline Thymoma & 7/12 (58.3\%) & 18/27 (66.7\%) & 0.723 \\
\hline Thymic hyperplasia & $3 / 12(25.0 \%)$ & 4/27 (14.8\%) & 0.654 \\
\hline Non-degraded thymus & $2 / 12(16.7 \%)$ & $5 / 27(18.5 \%)$ & 1.000 \\
\hline
\end{tabular}

Abbreviations: RNS Repetitive nerve stimulation, $y$ Year. Statistical analysis was performed by $x 2$ test and Fisher's exact test when necessary

0.906; $p=0.004$ ), positive facial nerve RNS (OR 3.496; 95\%CI 1.103-11.409; $p=0.033$ ) and positive axillary nerve RNS (OR 3.643; 95\%CI 1.012-13.049; $p=0.044$ ) significantly predicted GMG conversion except gender and thymic abnormalities. Further multivariate logistic regression analysis revealed that age at onset (OR 1.023, 95\% CI $1.006-1.041, p=0.007)$ and positive facial nerve RNS (OR $2.826,95 \% \mathrm{CI} 1.045-5.460, p=0.038$ ) were positively associated with generalized conversion. Moreover, an obviously negative association was found for disease duration (OR 0.603, 95\%CI 0.365-0.850, $p=0.019$ ) (Table 4).

\section{Discussion}

MG is a heterogeneous disease affected by ethnicity, gender, age at onset, disease duration and other factors. Generalized conversion is a typical feature for MG patients who initially present pure ocular symptoms, and approximately $90 \%$ of OMG patients from Caucasian populations might progress to GMG within the first 23 years $[1,2]$. However in this study enrolling OMG patients from Northwest China, only 38 of 223 patients (17.0\%) converted to GMG over a median disease course of 4 years, which was similar to previously reported

Table 3 Gender comparison of clinical features in 38 patients with generalized conversion

\begin{tabular}{|c|c|c|c|}
\hline Variables & Male & Female & $P$ value \\
\hline Patients with conversion, $n$ (\%) & $18(47.4)$ & $20(52.6)$ & - \\
\hline Age at onset (y), median (IQR) & $46(37.5-55)$ & $30.5(8-49.8)$ & 0.118 \\
\hline Time to generalization (y), median (IQR) & $0.8(0.2-2.0)$ & $0.9(0.3-2.9)$ & 0.692 \\
\hline \multicolumn{4}{|l|}{ Osserman classification, $n$} \\
\hline$\| \mathrm{A}$ & 8 & 13 & \\
\hline$\| \mathrm{B}$ & 9 & 6 & \\
\hline III & 1 & 1 & 0.512 \\
\hline
\end{tabular}

Abbreviations: $y$ Year, IQR Interquartile range. Statistical analysis was performed by Manne-Whitney $U$ test for age at onset and time to generalization, and by Fisher's exact test for Osserman classification between subgroups 

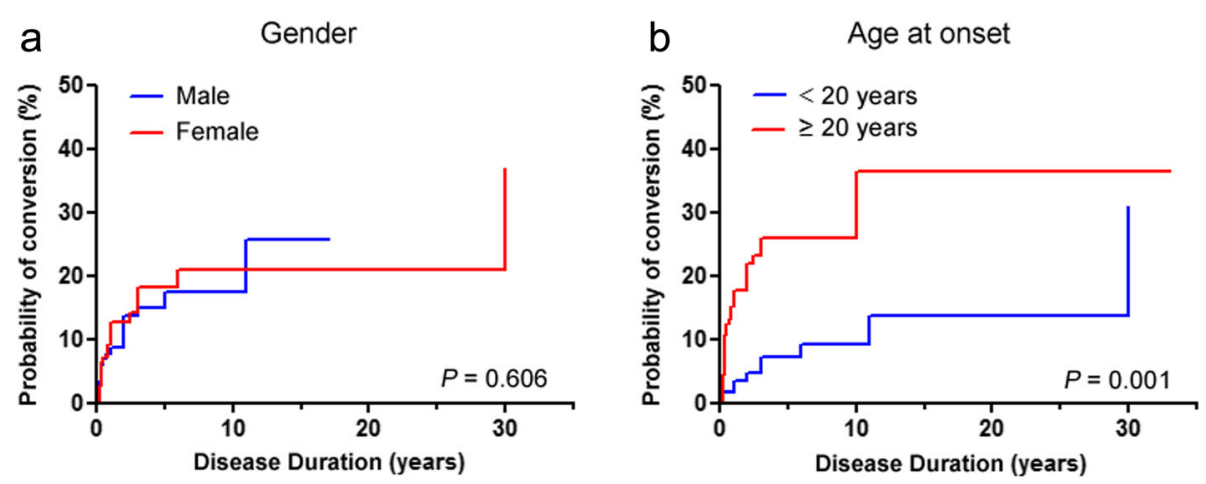

C Repetitive facial nerve stimulation

d Repetitive axillary nerve stimulation
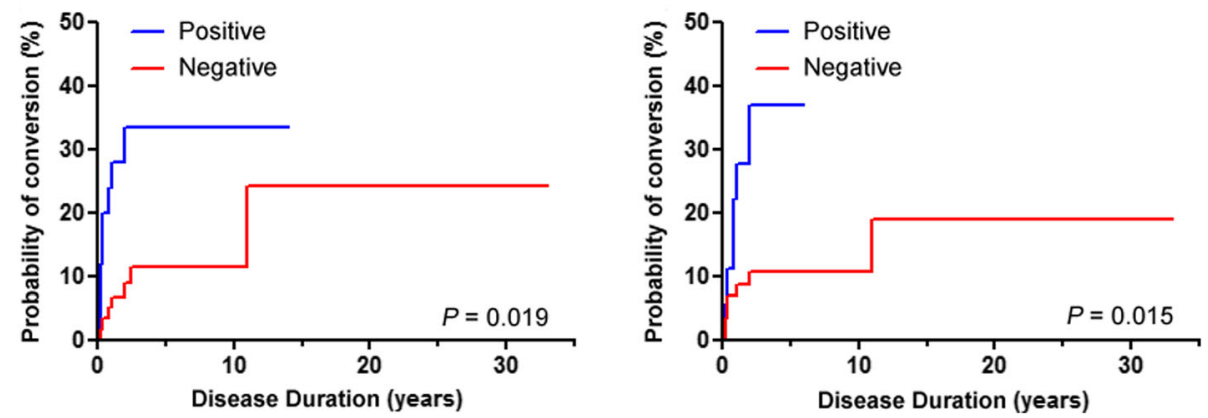

Fig. 1 a Kaplan-Meier curve depicting probability of conversion over time (years) from ocular to generalized myasthenia gravis between male and female patients. $\mathbf{b}$ Kaplan-Meier curve depicting probability of conversion over time (years) from ocular to generalized myasthenia gravis between juvenile-onset and adult patients. c Kaplan-Meier curve depicting probability of conversion over time (years) from ocular to generalized myasthenia gravis in patients with positive facial nerve RNS compared to those negative. $\mathbf{d}$ Kaplan-Meier curve depicting probability of conversion over time (years) from ocular to generalized myasthenia gravis in patients with positive axillary nerve RNS compared to those negative. $p<0.05$ indicates statistically significance

figures (11.4\%-29\%) in Asian populations from Southern China, Hong Kong, Korean and Singapore [6, 7, 12-14]. This discrepancy might be related to differential MG susceptibility at different ages. It is generally accepted that adults from Caucasian populations are more vulnerable to MG [15], but in Asian populations juvenile MG accounts for more than half of the MG patients [13]. Given that older age at onset has been demonstrated to correlate with higher risk of generalization [16, 17], the lower conversion rate in this study than in Caucasian population further highlights the potential role of ethnicity in the prevalence of OMG.

Several studies suggested that factors such as age at onset, AChR-Ab titers, positive rate of RNS, thymoma and immunosuppressive therapy were associated with generalization [6-9]. Immunosuppressive therapy has been given to OMG patients with intention to reduce the risk of secondary generalization $[2,4,18]$, but it is noted that a small proportion of patients still underwent generalized conversion [19] and the possible predictors remain unknown. In this study, we enrolled the OMG patients who received steroids and/or other immunosuppressants and found that $17.0 \%$ converted to GMG eventually. The conversion rate was consistent with previous studies where immunosuppressed and nonimmunosuppressed groups were compared $[6,7,19,20]$, and also suggested that the data was qualified for investigation of generalization of OMG under immunosuppressive therapy.

Age at onset has been identified as one of the risk factors for generalization of OMG. Prior studies showed that the conversion rate of juvenile-onset OMG fluctuated between 23 and $43 \%$ [3, 21], while that of adultonset OMG between 31 and 49\% [4, 22]. Kamarajah and Wang reported that late-onset was correlated with higher risk of generalization [16, 17]. In another study, patients were inclined to convert to GMG with increasing age at onset [2]. In this study, OMG patients under immunosuppressive treatments were divided into juvenile-onset OMG and adult-onset OMG subgroups. No significant differences were observed between the subgroups in clinical variables of interest except for axillary nerve RNS test. In Kaplan-Meier curve analysis, adult-onset OMG patients had a higher cumulative probability of conversion to GMG than juvenile-onset patients. Moreover, adult-onset patients had a shorter 


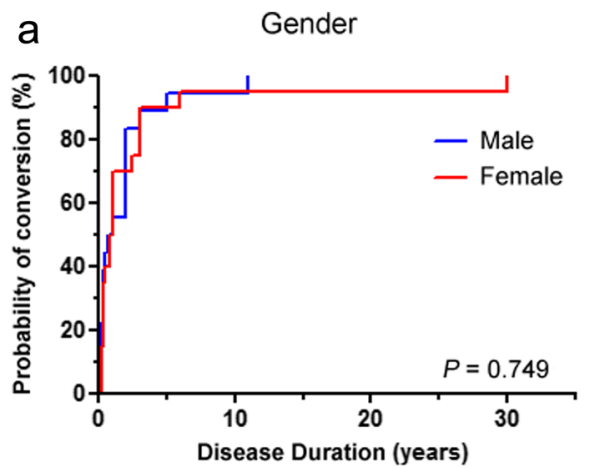

C Repetitive facial nerve stimulation

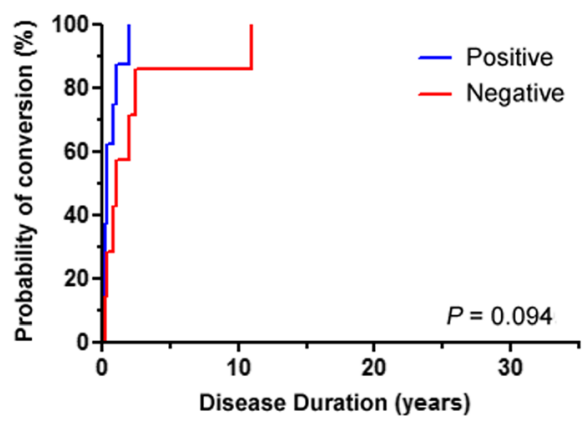

b Age at onset

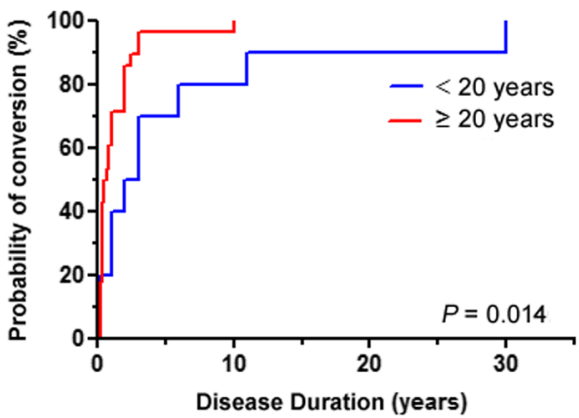

d Repetitive axillary nerve stimulation

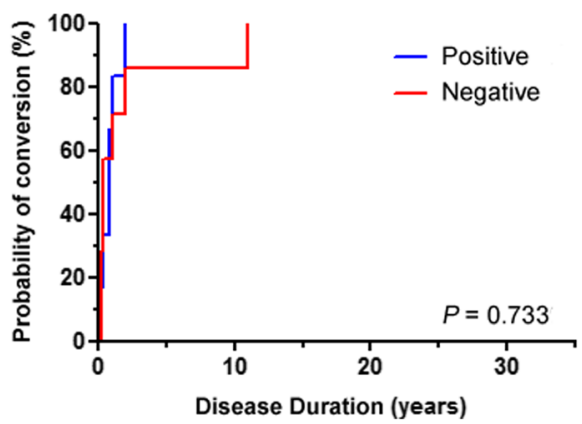

Fig. 2 a Kaplan-Meier curve depicting conversion tempo between male and female patients of 38 generalized. b Kaplan-Meier curve depicting conversion tempo between juvenile-onset and adult patients of 38 generalized. c Kaplan-Meier curve depicting conversion tempo in 38 generalized patients who had positive facial nerve RNS compared to those negative. $\mathbf{d}$ Kaplan-Meier curve depicting conversion tempo in 38 generalized patients who had positive axillary nerve RNS compared to those negative. $p<0.05$ indicates statistically significance

time to generalization than juvenile-onset patients. Further univariate and multivariate logistic regression analyses revealed that age at onset was positively associated with secondary generalization. Similar to previous studies $[2,16,17]$, our findings confirmed the predictive

Table 4 Logistic regression analysis of predictors of conversion to GMG

\begin{tabular}{lccc}
\hline & OR & \multicolumn{1}{c}{$95 \% \mathrm{Cl}$} & $P$ value \\
\hline Univariate logistic regression Variables & & \\
Gender & 1.252 & $0.621-2.537$ & 0.529 \\
Age at onset & 1.025 & $1.009-1.041$ & 0.002 \\
Disease duration & 0.783 & $0.651-0.906$ & 0.004 \\
Positive facial nerve RNS & 3.496 & $1.103-11.409$ & 0.033 \\
Positive axillary nerve RNS & 3.643 & $1.012-13.049$ & 0.044 \\
Thymic abnormalities & 2.259 & $0.949-5.202$ & 0.058 \\
Multivariate logistic regression Variables & & \\
Age at onset & 1.023 & $1.006-1.041$ & 0.007 \\
Disease duration & 0.603 & $0.365-0.850$ & 0.019 \\
Positive facial nerve RNS & 2.826 & $1.045-5.460$ & 0.038 \\
\hline
\end{tabular}

Abbreviations: RNS Repetitive nerve stimulation, OR Odds ratio, $\mathrm{Cl}$ Confidential interval value of age at onset in generalization of OMG under immunosuppressive therapy.

Positive RNS test is observed in a portion of OMG patients [23] and it is controversial whether positive RNS results predict secondary generalization. Some studies showed that positive RNS is associated with higher conversion rate $[6,7]$, whereas others did not support this $[1,4,6,24]$. In this study, positive facial RNS test was proved to be one predictor of secondary generalization. Although the association between axillary RNS test and risk of generalization was found in both Kaplan-Meier curve and univariate logistic regression analyses, but no clear association was evidenced by the multivariate logistic regression analysis, probably due to the limited number of patients who received axillary nerve RNS test. The predictive value of axillary nerve RNS test needs to be investigated in further studies with larger sample size and longer follow-up period.

Some scholars pointed out that positive AChR-Ab and abnormal single-fiber electromyography (SFEMG) tests in the early stages of the disease could predict more generalized conversion $[9,25,26]$, but the predictive values of these variables were not confirmed by other studies $[8,27]$. Unfortunately, we were unable to detect AChR- 
Ab titers and perform SFEMG due to restrictions of examination methods, but this variable was strongly recommend to be included in future studies. Thymic abnormalities, especially thymoma has been considered one predictor of secondary generalization [8]. However in our cohort, thymic abnormality was excluded as a potential predictor during logistic regression analysis. This might be interpreted to some extent by early application of immunosuppressive therapy since it could weaken or eliminate the effect of thymic abnormalities in promoting generalized conversion. In addition, studies have shown that the longer disease duration the lower conversion risk of OMG [19, 26, 28]. By multivariate logistic regression analysis, we found that disease duration was negatively associated with generalization in the OMG patients receiving immunosuppressive therapy. The similar findings suggested that immunosuppressive treatments could not hamper the effect of disease duration on secondary generalization.

There are some limitations in this retrospective study. Restricted clinical condition and examination methods, unwillingness of partial patients and missing data from medical records resulted in small sample size of clinical variables, especially RNS tests in our cohort, but the percentage of patients who entered the final statistical analysis and of those with positive RNS tests were comparable to other studies [7, 24, 29]. This supported that the conclusions from statistical analysis in this study were reliable. In the future, prospective studies with rigorous design and complete data as well as with larger sample size and longer follow-up period are recommended to obtain more convincing results and conclusions.

\section{Conclusions}

Immunosuppressive therapy can reduce, but not avoid generalization of OMG. Among the patients who receive immunosuppressive treatments, age at onset, disease duration and positive facial nerve RNS are identified as the predictive factors of generalized conversion. Adultonset, shorter disease duration and positive facial nerve RNS indicate the higher risk of secondary generalization. Further prospective studies with larger sample size and longer follow-up period are needed to corroborate the conclusions pertaining to this study.

\section{Abbreviations}

MG: Myasthenia gravis; OMG: Ocular myasthenia gravis; GMG: Generalized myasthenia gravis; RNS: Repetitive nerve stimulation; OR: Odds ratio; $\mathrm{Cl}$ : Confidence interval; AChR-Ab: Anti-acetylcholine receptor antibody; IQR: Interquartile range; y: Year

\section{Acknowledgements}

The authors appreciate Dr. Hui Liu for his excellent work in revising and polishing the manuscript.

\section{Authors' contributions}

Study concept and drafting of the manuscript: JQD, SJZ, KXR. Collection of data and statistical analysis: DD, FW, MZ. Revision of manuscript for important intellectual content: HZL, ZYL. Study concept and design, critical revision of the manuscript for important intellectual content: JG. All authors have read and approved of the final version of the manuscript.

\section{Funding}

This study was funded by the National Natural Science Foundation of China (Grant No. 81571218). The funding body had no role in the design of the study and collection, analysis, and interpretation of data and in writing the manuscript.

\section{Availability of data and materials}

The data sets in this study are available from the corresponding author on reasonable request.

\section{Ethics approval and consent to participate}

This study protocols were approved by the ethics committees of Tangdu Hospital, Fourth Military Medical University. Written informed consent was obtained from all patients participating in this study.

Consent for publication

Not applicable.

Competing interests

The authors declare that they have no competing interests.

\section{Author details}

'Department of Neurology, Tangdu Hospital, Fourth Military Medical University, Xi'an 710038, Shaanxi Province, China. ${ }^{2}$ Intensive Care Unit, Xi'an Fourth Hospital, Xi'an 710004, Shaanxi Province, China. ${ }^{3}$ Department of Neurology, Xi'an Children's Hospital, Xi'an 710003, Shaanxi Province, China.

Received: 21 January 2020 Accepted: 26 May 2020

Published online: 11 June 2020

References

1. Bever CT Jr, Aquino AV, Penn AS, Lovelace RE, Rowland LP. Prognosis of ocular myasthenia. Ann Neurol. 1983;14(5):516-9.

2. Kupersmith MJ, Latkany R, Homel P. Development of generalized disease at 2 years in patients with ocular myasthenia gravis. Arch Neurol. 2003;60(2): 243-8.

3. Remes-Troche JM, Téllez-Zenteno JF, Estañol B, Garduño-Espinoza J, GarcíaRamos G. Thymectomy in myasthenia gravis: response, complications, and associated conditions. Arch Med Res. 2002:33(6):545-51.

4. Sommer N, Sigg B, Melms A, Weller M, Schepelmann K, Herzau V, et al. Ocular myasthenia gravis: response to long-term immunosuppressive treatment. J Neurol Neurosurg Psychiatry. 1997;62(2):156-62.

5. Romi F, Gilhus NE, Aarli JA. Myasthenia gravis: clinical, immunological, and therapeutic advances. Acta Neurol Scand. 2005;111(2):134-41.

6. Hong YH, Kwon SB, Kim BJ, Kim BJ, Kim SH, Kim JK, et al. Prognosis of ocular myasthenia in Korea: a retrospective multicenter analysis of 202 patients. Neurol Sci. 2008;273(1-2):10-4.

7. Teo KY, Tow SL, Haaland B, Gosavi TD, Jing-Liang L, Yew Long LO, et al. Low conversion rate of ocular to generalized myasthenia gravis in Singapore. Muscle Nerve. 2018:57(5):756-60.

8. Li F, Hotter B, Swierzy M, Ismail M, Meisel A, Rückert JC. Generalization after ocular onset in myasthenia gravis: a case series in Germany. J Neurol. 2018; 265(12):2773-82

9. Allen JA, Scala S, Jones HR. Ocular myasthenia gravis in a senior population: diagnosis, therapy, and prognosis. Muscle Nerve. 2010;41(3):379-84.

10. Leeamornsiri S, Chirapapaisan N, Chuenkongkaew W. Clinical profiles of Thai patients with ocular myasthenia gravis in Siriraj hospital. J Med Assoc Thail. 2011;94(9):1117-21.

11. Juel VC, Massey JM. Myasthenia gravis. Orphanet J Rare Dis. 2007:2:44

12. Huang $X, L i Y$, Feng H, Chen P, Liu W. Clinical characteristics of juvenile myasthenia gravis in southern China. Front Neurol. 2018:9:77.

13. Gui M, Luo X, Lin J, Li Y, Zhang M, Zhang X, et al. Long-term outcome of 424 childhood-onset myasthenia gravis patients. J Neurol. 2015;262(4):823-30. 
14. Wong V, Hawkins BR, Yu YL. Myasthenia gravis in Hong Kong Chinese. 2 Paediatric disease. Acta Neurol Scand. 1992;86(1):68-72.

15. Phillips LH 2nd, Torner JC, Anderson MS, Cox GM. The epidemiology of myasthenia gravis in central and western Virginia. Neurology. 1992;42(10): 1888-93.

16. Kamarajah SK, Sadalage G, Palmer J, Carley H, Maddison P, Sivaguru A. Ocular presentation of myasthenia gravis: a natural history cohort. Muscle Nerve. 2018:57(4):622-7.

17. Wang LL, Zhang Y, He ML. Clinical features and prognosis of ocular myasthenia gravis patients with different phenotypes. Chin Med J. 2015; 128(19):2682-4

18. Zach H, Cetin H, Hilger E, Paul A, Wuschitz B, Jung R, et al. The effect of early prednisolone treatment on the generalization rate in ocular myasthenia gravis. Eur J Neurol. 2013;20(4):708-13.

19. Nagia L, Lemos J, Abusamra K, Cornblath WT, Eggenberger ER. Prognosis of ocular myasthenia gravis: retrospective multicenter analysis. Ophthalmology. 2015;122(7):1517-21.

20. Monsul NT, Patwa HS, Knorr AM, Lesser RL, Goldstein JM. The effect of prednisone on the progression from ocular to generalized myasthenia gravis. J Neurol Sci. 2004;217(2):131-3.

21. Pineles SL, Avery RA, Moss HE, Finkel R, Blinman T, Kaiser L, et al. Visual and systemic outcomes in pediatric ocular myasthenia gravis. Am J Ophthalmol. 2010;150(4):453-9.

22. McCreery KM, Hussein MA, Lee AG, Paysse EA, Chandran R, Coats DK. Major review: the clinical spectrum of pediatric myasthenia gravis: blepharoptosis, ophthalmoplegia and strabismus. A report of 14 cases. Binocul Vis Strabismus Q. 2002;17(3):181-6.

23. Fan L, Ma S, Yang Y, Yan Z, Li J, Li Z. Clinical differences of early and lateonset myasthenia gravis in 985 patients. Neurol Res. 2019;41(1):45-51.

24. Mazzoli M, Ariatti A, Valzania F, Kaleci S, Tondelli M, Nichelli PF, et al. Factors affecting outcome in ocular myasthenia gravis. Int I Neurosci. 2018;128(1): $15-24$.

25. Galassi G, Mazzoli M, Ariatti A, Kaleci S, Valzania F, Nichelli PF. Antibody profile may predict outcome in ocular myasthenia gravis. Acta Neurol Belg. 2018;118(3):435-43.

26. Hendricks TM, Bhatti MT, Hodge DO, Chen JJ. Incidence, epidemiology, and transformation of ocular myasthenia gravis: a population-based study. Am J Ophthalmol. 2019;205:99-105.

27. Lai $\mathrm{CH}$, Tseng HF. Nationwide population-based epidemiological study of myasthenia gravis in Taiwan. Neuroepidemiology. 2010;35(1):66-71.

28. Grob D, Brunner N, Namba T, Pagala M. Lifetime course of myasthenia gravis. Muscle Nerve. 2008;37(2):141-9.

29. Rodríguez J, Dimitrova NA, Dimitrov GV, Gila L. Shape variability of potentials recorded by a single-fiber electrode and its effect on jitter estimation. Ann Biomed Eng. 2011;39(2):812-23.

\section{Publisher's Note}

Springer Nature remains neutral with regard to jurisdictional claims in published maps and institutional affiliations.

Ready to submit your research? Choose BMC and benefit from:

- fast, convenient online submission

- thorough peer review by experienced researchers in your field

- rapid publication on acceptance

- support for research data, including large and complex data types

- gold Open Access which fosters wider collaboration and increased citations

- maximum visibility for your research: over $100 \mathrm{M}$ website views per year

At $\mathrm{BMC}$, research is always in progress.

Learn more biomedcentral.com/submissions 